\title{
Gain and Stability Models for HBT Grid Amplifiers
}

\author{
Cheh-Ming Liu*1, Emilio A. Sovero ${ }^{2}$, Michael P. De Lisio ${ }^{1}$, \\ Alina Moussessian ${ }^{1}$, James J. Rosenberg ${ }^{3}$, and David B. Rutledge ${ }^{1}$ \\ ${ }^{1}$ Department of Electrical Engineering, California Institute of Technology, Pasadena, CA 91125 \\ ${ }^{2}$ Rockwell International Corporation, 1049 Camino Dos Rios, Thousand Oaks, CA 91385 \\ 3 Engineering Department, Harvey Mudd College, Claremont, CA 91711.
}

Abstract-A 16-element heterojunction-bipolar-transistor (HBT) grid amplifier has been fabricated with a peak gain of $11 \mathrm{~dB}$ at $9.9 \mathrm{GHz}$ with a $3-\mathrm{dB}$ bandwidth of $350 \mathrm{MHz}$. Here we report a model of gain analysis for the grid and give a comparison of the measurement and theory. Measured patterns gives evidence of a common-mode oscillation. A stability model for the common-mode oscillation is developed. Based on the stability model, a lumped capacitor gives suitable phase shift of the circular function, thus stabilizing the grid. A second 16-element grid was fabricated, using this theory, with improved stability.

\section{INTRODUCTION}

A variety of spatial power combining amplifiers have been explored recently[1-6]. The development of quasi-optical amplifiers combine powers in free space and eliminate losses associated with waveguide walls and feed networks $[7,8]$. By the quasi-optical technology, grid amplifiers demonstrated gains of $11 \mathrm{~dB}$ at C-band[1] and $10 \mathrm{~dB}$ at X-band[2]. However, these grids were designed empirically. In this paper, we report models of gain and stability for the grid amplifier. Based on the models, two 16-element grids were fabricated on Duroid substrates with a dielectric constant of 10.8 . The active devices were HBT amplifier chips[2].

\section{Grid-Amplifier Gain Model}

A grid amplifier is composed of many unit cells uniformly distributed on a dielectric substrate. The behavior of a grid amplifier is characterized by the unit cell. A unit cell of the grid amplifier is shown in Fig. 1a. In the unit cell, an HBT differential pair is located in the center and biased by horizontal

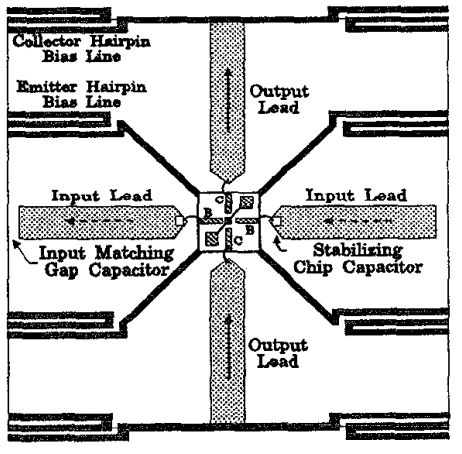

(a)

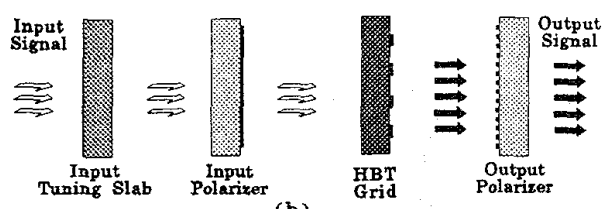

(b)

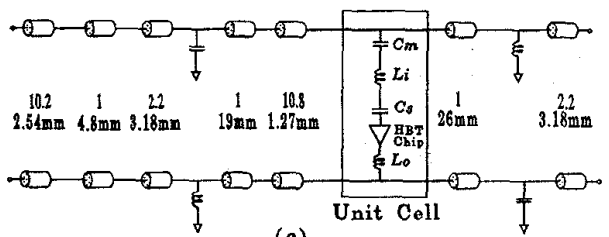

(c)

Figure 1. (a) Unit cell. The arrows indicate the current flows. In fabrication, the unit cell is $8 \mathrm{~mm}$ $x 8 \mathrm{~mm}$. The input and output leads are $0.8 \mathrm{~mm}$ wide. The gap of the input matching capacitor is $0.1 \mathrm{~mm}$. (b) Side view of grid amplifier. (c) Transmission-line equivalent-circuit model for gain analysis. The numerical values are the dielectric constants and thicknesses of dielectric layers and air gaps. 


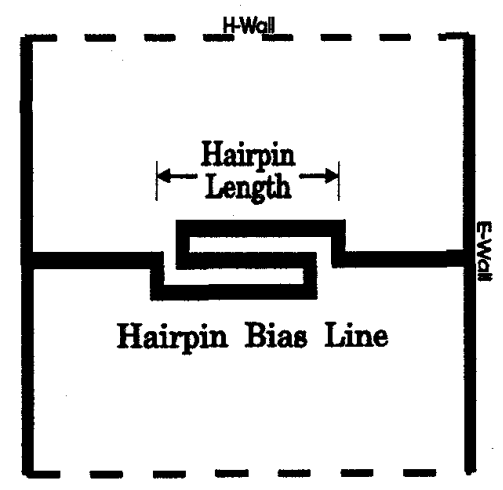

(a)

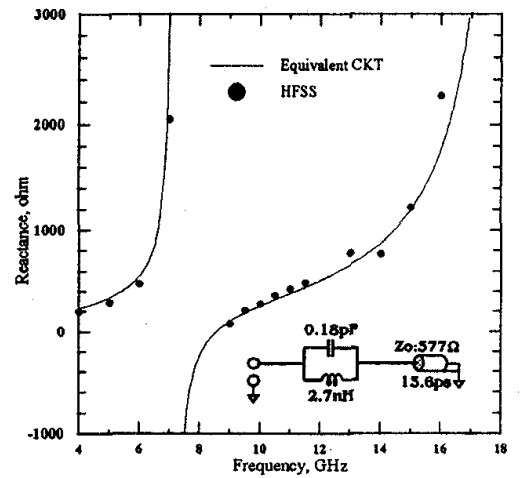

(b)

Figure 2. (a) The hairpin bias line. Boundary conditions are imposed by grid symmetry. Unit cell is $8 \mathrm{~mm} \times 8 \mathrm{~mm}$. The line is $0.1 \mathrm{~mm}$ wide and the hairpin length is $3.1 \mathrm{~mm}$. Resonant frequency is determined by hairpin length. (b) Reactance of hairpin bias line.

hairpin bias lines. The horizontally polarized input signal is received by the input leads, amplified, and reradiated through the vertical output lead with vertical polarization. Based on the analysis of the unit cell, a grid amplifier (Fig. 1b) is represented by a transmission-line equivalent-circuit model (Fig. 1c). Dielectric layers and air gaps are represented by transmission lines. In the model, the upper sequence of transmission lines is horizontally polarized input. Similarly, the lower sequence of transmission lines is vertically polarized output. The unit cell links the input and output. The input polarizer acts as a capacitor for input and an inductor for output. The output polarizer acts as an inductor for input and a capacitor for output. In addition, there is a dielectric input tuning slab that is represented by a section of transmission line. For the unit cell, the input and output leads are represented by inductors, $L_{i}$ and $L_{o}$. The input matching capacitor, $C_{m}$, is to match the inductive impedance of the input lead. The stabilizing capacitor, $C_{s}$, improves the stability of the grid amplifier, and will be described in the following section.

The hairpin bias lines are designed to have high impedance at the design frequency (Fig. 2a). The length is about a quarter of wavelength for material with mean dielectric constant at design frequency. The structure was analyzed by Hewlett-Packard High-Frequency-Structure-Simulator (HFSS), and results are shown in Fig. 2b. The solid line shows the results of fitted parameters. For this structure, the resonant frequency is $7.2 \mathrm{GHz}$. By scaling the length, we could shift the resonant frequency close to the design frequency. In our grid, the hairpin length is $2.1 \mathrm{~mm}$.

A 16-element HBT grid was fabricated on a Duroid substrate with dielectric constant 10.8 and the gain is measured in far-field measurement[2]. The maximum gain is $11 \mathrm{~dB}$ at $9.9 \mathrm{GHz}$ and the 3- $\mathrm{dB}$ bandwidth is $350 \mathrm{MHz}$. A comparison of the measured and theoretical gains is shown in Fig. 3a.

\section{GRID-AMPLIFIER STABILITY MODEL}

Without the stabilizing capacitor, the grid oscillates at $7.8 \mathrm{GHz}$ as the bias current increases to $2 \mathrm{~mA}$ per transistor. If we short out the input matehing gap capacitors (Fig. 1a) with bond wires, the oscillation frequency shifts to $4.8 \mathrm{GHz}$. However, the oscillation is not sensitive to the substrate thickness and the polarizer positions. The greatest power density is in the plane of board and with polarization parallel to the plane. The azimuth oscillation pattern is shown in Fig. $3 \mathrm{~b}$. The significant peak on the emitter bias end characterizes the oscillation a common-mode source excited by vertically distributed currents, the collector currents.

A model of the common-mode oscillation is shown in Fig. 4. The input and output leads are 


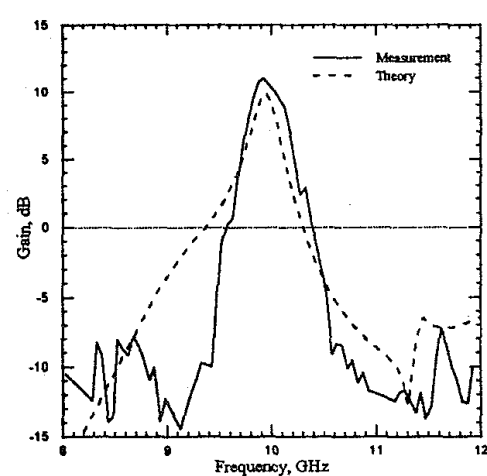

(a)

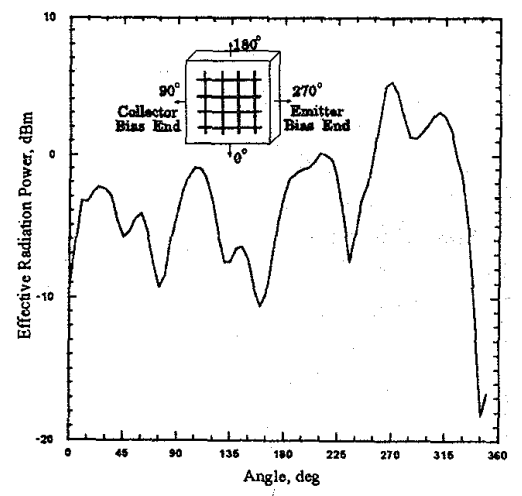

(b)

Figure 3. (a) Gain of grid amplifier. The measured peak gain is $11 \mathrm{~dB}$ at $9.9 \mathrm{GHz}$. The simulated peak gain is $10 \mathrm{~dB}$ at $10 \mathrm{GHz}$. In simulation, the inductance of the input and output leads are 1.7 $\mathrm{nH}$, derived from a method-of-moment calculation, and the input matching capacitor is $0.27 \mathrm{pF}$, derived from an HFSS simulation. (b) Azimuth oscillation patterns. Measured polarization is in the plane of board. The peak power in the plane of board is $6 \mathrm{~dB}$ higher than for the normal plane.

represented by transmission lines, rather than inductors in the gain model. Fig. $5 \mathrm{a}$ shows the plots of the circular function, which is the reflection coefficient of the circulator port[9]. In Martinez' theory, for small signal analysis, the circuit oscillates at the frequency where the magnitude of the circular function is larger than unity and the phase of the circular function is zero. The impedance of transmission line is chosen to give an oscillation frequency of the observed oscillation. To stabilize the grid, a lumped component, stabilizing capacitor, is added at the base of the HBT. This stabilizing capacitor offers a positive phase shift to the circular function. The circuit is now stable, with a phase margin of $23^{\circ}$ and a gain margin of $1.6 \mathrm{~dB}$.

To test this model, another grid was fabricated with the stabilizing capacitors. The plot of gain versus bias voltage is shown in Fig. 5b. No oscillation was observed. When biased above 17 volts, the grid became hot. In the measurement, the maximum gain is $8.5 \mathrm{~dB}$ at 17 volts and the bias current is $11 \mathrm{~mA}$ per transistor. This bias current is two times as high as the bias current of the previous grid. The stabilizing capacitor reduces the gain by $2.5 \mathrm{~dB}$, but should give the grid high maximum output power.

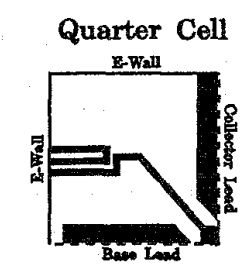

(a)

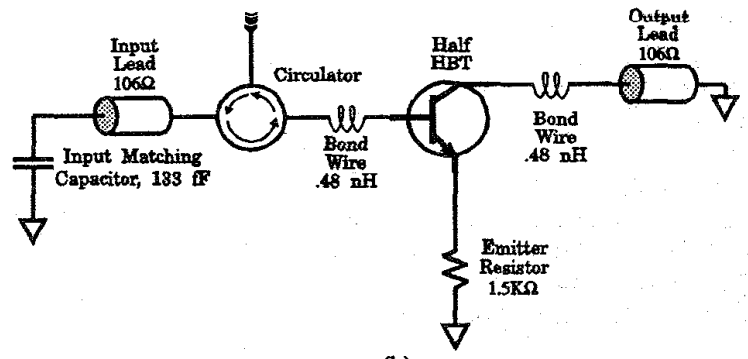

(b)

Figure 4. (a) Quarter cell (b) Equivalent-circuit model for common-mode oscillation. Boundary conditions are imposed by grid symmetry and common-mode operation of HBT pair. 


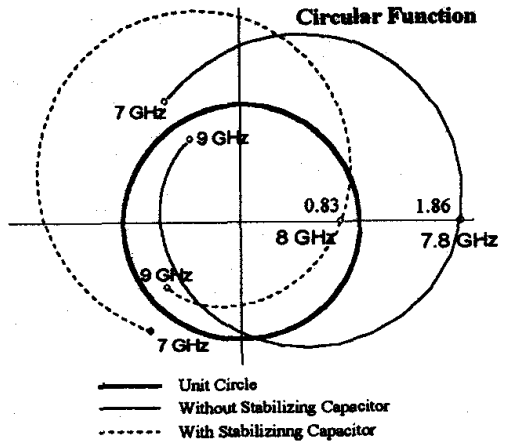

(a)

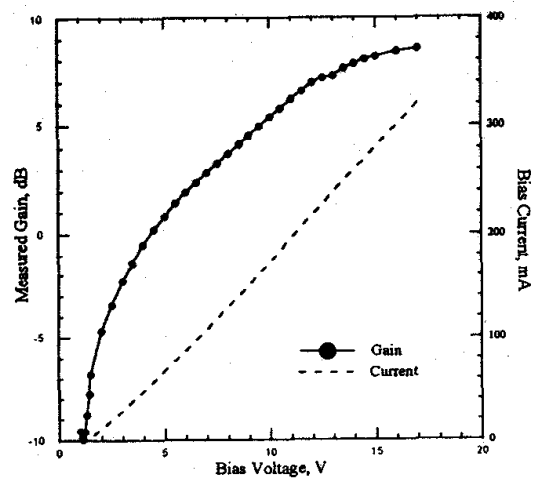

(b)

Figure 5. (a) Circular function loci. The frequency is swept from $7 \mathrm{GHz}$ to $9 \mathrm{GHz}$. The stabilizing capacitor is $0.1 \mathrm{pF}$. (b) Gain and bias current versus bias voltage at $10 \mathrm{GHz}$.

\section{CONCLUSION}

We have reported a gain model and demonstrated a hairpin-bias-line HBT grid with a peak gain of $11 \mathrm{~dB}$ at $9.9 \mathrm{GHz}$. For stability, we have developed a model for common-mode oscillation from the oscillation patterns. Consequently, a highly stable grid amplifier is achieved.

\section{ACKNOWLEDGMENTS}

The authors would like to appreciate the support of Air Force Material Command/Rome Laboratory and Army Research Office.

\section{REFERENCE}

[1] M. Kim, J. Rosenberg, R. Smith, R. Weikle, II, J. Hacker, M. De lisio, and D. Rutledge, "A Grid Amplifier," IEEE Microwave Guided Wave Lett. , vol. 1, pp. 322-324, 1991.

[2] M. Kim, E. Sovero, J. Hacker, M. De lisio, J.-C. Chiao, S. Li, D. Gagnon, J. Rosenberg, and B. Rutledge, "A 100-Element grid Amplifier," IEEE Trans. Microwave Theory Tech., vol. 41, pp. 1762-1771, October 1993.

[3] C.-Y. Chi and G. Rebeiz, "A Quasi-Optical Amplifier," IEEE Microwave Guided Wave Lett., vol. 3, pp. 164-166, June 1993.

[4] J. Benet, A. Perkons, S. Wong, and A. Zaman, "Spatial Power Combining for Millimeter-wave Solid State Amplifier," IEEE MTT-S Int. Microwave Symp. Dig., pp. 619-622, 1993.

[5] T. Mader, J. Schoenberg, L. Harmon, and Z. Popovic , "Planar MESFET Transmission Wave Amplifier," Electronic Lett., vol. 29, pp. 1699-1701, September 1993.

[6] N. Sheth, T. Ivanov, A. Balasubramaniyan, and A. Mortazawi, "A Nine HEMT Spatial Amplifier," IEEE MTT-S Int. Microwave Symp. Dig., 1994, pp. 1239-1242.

[7] D. Rutledge, J. Hacker, M. Kim, R. Weikle, II, R. Smith, and E. Sovero, "Oscillator and Amplifier Grids," IEEE MTT-S Int. Microwave Symp. Dig., 1992, pp. 815-817.

[8] R. York, "Quasi-Optical Power Combining Techniques," to be published in SPIE Critical Reviews of Emerging Technologies, J.Wiltse, ed., 1994.

[9] R. Martinez and R. Compton, "A General Approach for the S-parameter Design of Oscillator with 1 and 2-Port Active Devices," IEEE Trans. Microwave Theory Tech., vol. 40, pp. 569-574, March 1992. 